\title{
ENTREVISTA A ROSSANA CAMPO ${ }^{1}$
}

\author{
Pablo García Valdés 2
}

Rossana Campo nació en Génova, en 1963, en el seno de una familia napolitana que emigra al norte en los años del boom económico. Estudió Letras en la Universidad de Génova, donde entra en contacto con el profesor Edoardo Sanguineti, poeta, crítico y teórico literario, máximo exponente del Gruppo 63, un literato que ha sido el abanderado de la Neoavanguardia italiana.

El influjo de este autor en sus libros se aprecia sobretodo en las primeras novelas, de índole más experimental. Estas influencias se hacen visibles en el abandono de las formas tradicionales del lenguaje, sustituyéndolas por nuevas estructuras y temáticas renovadas. Con ello, Rossana Campo pretende desarrollar una literatura fresca e innovadora que pudiese estar al nivel de otras literaturas europeas, abandonando la literatura italiana anclada en formas y temáticas del pasado. Además de los miembros de este movimiento literario, la italiana se ha visto influenciada por la obra de Henry Miller y de Gertrude Stein, siendo autores capitales en la configuración de sus temáticas y de su estilo.

El exordio literario de Rossana Campo tiene lugar en 1992, aunque previamente ya había realizado pequeñas publicaciones conjuntas. La autora ha publicado las siguientes novelas: In principio erano le mutande (1992), Il pieno di super (1993), Mai sentita così bene (1995), L'attore americano (1997), Il matrimonio di María (1998), Mentre la mia bella dorme (1999), Sono pazza di te (2001), L'uomo che non ho sposato (2003), Duro come l'amore (2005), Più forte di me (2007), Lezioni di arabo (2010), Felice per quello che sei. Confessioni di una buddista emotiva (2012), Il posto delle donne (2013), Fare l'amore (2014) y, más recientemente, Dove troverete un altro padre come il mio (2015).

\footnotetext{
1 Fecha de recepción: 01/07/2015.

2 Doctorando, Departamento de Filología Clásica y Románica, Universidad de Oviedo; $\square$ pablogarvaldes@gmail.com.
} 
Además, ha publicado dos libros infantiles: La gemella buona e la gemella cattiva (2000) y Piccoli Budda (2013), y un libro de pintura: L'arte soppianta tutti gli altri enzimi (2012).

Rossana Campo propone la escritura y la literatura como un medio de evasión mediante el cual las personas tienen el derecho de crear un espacio propio para poder expresarse con total libertad, porque, según relata en uno de sus libros, cuando una lee un libro quiere alejarse de las cosas feas de la vida. Por este motivo, la autora analiza y describe a través de sus heroinas una determinada realidad social. A partir de los temas predilectos de la literatura femenina, como son el amor, el erotismo y las relaciones personales, Rossana Campo pretende romper el modelo tradicional de feminidad establecido por la sociedad patriarcal. Sus heroínas constituyen un caleidoscopio de personalidades que constituyen un reflejo del pensamiento y de los problemas que afligen a las mujeres en las diversas etapas de la vida: desde el descubrimeinto del amor, hasta el miedo a la soledad en la edad adulta.

El concepto de amor y de erotismo se ve en la obra de Rossana Campo desde una óptica contemporánea y feminista. La escritora habla abiertamente en sus libros del amor sin compromiso, sin dependencia; también propone una concepción libre del sexo y manifiesta su rechazo hacia la insatisfacción sexual de las mujeres, defendiendo la libertad en el matrimonio, el amor homosexual, etc. Temáticas que, pocos años antes, habían sido tabú para las mujeres.

Es aquí donde encontramos la importancia de tipo de estudios, donde se da luz y se pone de relieve los valores que Rossana Campo pretende transmitir con su obra. A modo de resumen, sobre el tema del amor, la escritora habla del enamoramiento, de la felicidad, pero también de la falta de amor, de la soledad; sobre el erotismo se puede afirmar que aún falta una cultura sobre la sexualidad femenina, puesto que en sus libros intenta conseguir un cambio en la concepción tradicional de la sociedad. Según los textos de la escritora, existe un fuerte mecanismo cultural por el cual no se ve la importancia del intercambio, sino de la posesión, $y$, por tanto, rechaza el campromiso como fórmula para obtener una mayor independencia y libertad para la mujer. Como podemos comprobar, 
todas estas temáticas tienen como nexo en común la libertad de las mujeres en todos los campos (en el amor, en las relaciones de pareja y en su sexualidad). Por todo ello, los libros de Rossana Campo pueden considerarse un punto de referencia para los estudios de género, puesto que hacen un crítica voraz a la visión tradicional de la sociedad patriarcal hacia las mujeres.

Gracias a la amabilidad y colaboración de la escritora se ha podido realizar la siguiente entrevista, que, sin lugar a duda, es de gran ayuda para aclarar algunos puntos esenciales de la vita, de los influjos y de las temáticas abordadas en la obra de la escritora y, así, comprender la importancia que estas tienen en sus libros.

\section{Da dove viene la sua vocazione per la scrittura? Cosa significa per Lei dedicarsi alla scrittura al giorno d'oggi?}

Ho sempre scritto, fin da ragazzina. Per quanto mi possa ricordare, nella mia vita ci sono stati sempre libri da leggere e da scrivere. Da dove nasce una passione? Difficile da dire, io non sono nata in una famiglia di intellettuali, i miei non hanno mai letto granché, per me invece, fin da bambina, il mondo delle favole era un posto che mi attirava come una calamita, un luogo che mi sembrava più interessante, più emozionante e più vero della realtà quotidiana. Così ho continuato a farlo e oggi che ho cinquant'anni continuo ogni giorno con la stessa passione a leggere e scrivere. Quindi non so 'cosa significa oggi scrivere', è qualcosa di naturale per me, un po' come respirare.

\section{Essendo stata allieva di Sanguineti all'Università di Genova, in che modo ha influenzato questo autore la Sua opera?}

Conoscere i romanzi e le poesie di Sanguineti e di tutti gli altri scrittori della cosiddetta Neoavanguardia letteraria italiana (cioè del Gruppo '63) è stata un'esperienza straordinaria, ho scoperto che anche con la lingua italiana si potevano sperimentare linguaggi e strutture nuove, è stata la scoperta che la letteratura italiana poteva essere 
interessante quanto quella inglese o americana o francese, per dire, mentre prima gli scrittori italiani che avevo letto mi sembrava che avessero sempre come un odore di muffa, che deriva dal cattolicesimo, forse, o dall'ipocrisia scolastica. Nei loro libri erano sempre un po' ingessati, fasulli, sempre poco liberi e poco avventurosi. Nella lingua usata come nelle storie raccontate. Come mi ha insegnato Sanguineti, il linguaggio è già ideologia, e quindi ad una lingua vecchia e conformista corrisponde un immaginario simile!

\section{Cosa ne pensa della situazione della narrativa femminile in Italia?}

Ma non saprei dire, so che ci sono scrittrici interessanti e altre che mi evocano quello che dicevo sopra a proposito di alcuni scrittori italiani, le trovo poco libere, poco avventurose. Come se scrivessero esattamente quello che ci si aspetta che scrivano.

\section{È difficile per una donna parlare in Italia dell'amore libero o di sesso?}

Per me non lo è, dato che scrivere è un lavoro di conoscenza di se stessi e del mondo, delle relazioni eccetera, se si fa un percorso di sincerità dentro di sé credo non si posso fingere quando poi si comincia a scrivere.

\section{Quasi tutte le protagoniste dei Suoi libri hanno una particolare visione dell'amore, dell'erotismo e delle relazioni umane. Cos'è per Rossana Campo l'amore?}

L'amore può essere un grande inganno, una perdita di energia, perdita di libertà e di consapevolezza. specie per noi donne che siamo state educate nei secoli a mettere tutte le nostre aspettative in un marito, può essere una fregatura se si vive come attesa dell'uomo che ti renderà felice. C'è poi un altro tipo di amore che è fatto di libertà e di scoperta di sè in una relazione che è molto più interessante. 
Il racconto dettagliato delle relazioni intime è un punto fondamentale di opere come Duro come l'amore o Lezioni di arabo. Come si deve interpretare l'erotismo nei Suoi romanzi?

Ognuno lo interpreti come crede! a me interessa seguire le mie eroine anche in camera da letto, mi piace sentire cosa provano quando fanno l'amore, quando si lasciano andare o hanno un orgasmo. Trovo interessanti gli scrittori che si addentrano anche nella vita erotica dei loro personaggi. Non capisco perché sappiamo tutto di come un personaggio mangia, si veste, cammina, lavora, e poi ci si debba fermare fuori dalla camera da letto.

Secondo Lei, qual è o quale deve essere il rapporto tra amore e sesso?

Ah non lo so. Credo sia interessante esplorare tutto quanto, è ciò che rende interessante il fatto di essere vivi, dunque a me personalmente interessa vivere e raccontare tutto, l'amore, il sesso con chi ami, il sesso da soli, il sesso vissuto solo per godimento carnale, tutto è interessante se vissuto con consapevolezza.

Dalla lettura dei Suoi romanzi si vede chiaramente che tra l'amante e il marito, Lei preferisce l'amante. Che ruolo avrebbe per Lei questi? Crede che sia fallita l’istituzione del matrimonio?

Non ho opinioni al riguardo, ripeto, tutto è interessante quando è vissuto e narrato in modo consapevole, quando non ci aspettiamo da fuori la soluzione ai nostri problemi, o che la felicità ci arrivi da qualcosa o qualcuno di esterno. Abbiamo bisogno di sperimentare, così nelle diverse fasi della vita proviamo: matrimoni, amanti, amici, persone dello stesso sesso, di sesso diverso... tutto è interessante! 
Le protagoniste vengono a volte concepite come eroine oppure antieroine. Come sono state create? Cosa c'è di Rossana Campo in tutte quante?

In ogni personaggio c'è la vita come io la sento. Io sento ciò che le mie eroine sentono, questo non significa ovviamente che ciò che racconto è la cronaca della mia vita!

Il conflitto interno, l'abbandono, il disadattamento... sono caratteristiche proprie delle protagoniste, come si deve combattere contro questi sentimenti? Si può arrivare alla felicità?

La felicità è lo scopo della vita. L'ho sempre pensato, e quando vent'anni fa ho incontrato il pensiero buddista ho scoperto che lo pensava anche un certo signor Siddharta, in India, circa 2500 anni fa. Noi possiamo diventare felici abbracciando tutto quello che sentiamo, che proviamo, anche le nostre parti più fragili, disadattate, sofferenti. Abbracciando le nostre parti randagie. E pian piano facendo così, imparando ad amarci davvero, impariamo a prenderci cura sinceramente anche di tutti gli altri esseri. Non nel senso di sacrificarci per gli altri, ma cercando l'armonia. Non è un caso se ho scritto un libro che s'intitola: Felice per quello che sei. Confessioni di una buddista emotiva. 